\title{
Is treatment-resistant depression a useful concept?
}

\author{
Gin S Malhi, ${ }^{1,2,3}$ Yulisha Byrow ${ }^{1,2}$
}

${ }^{1}$ Department of Psychiatry, CADE Clinic, Royal North Shore Hospital, Sydney, New South Wales, Australia; ${ }^{2}$ Discipline of Psychiatry, Sydney Medical School, University of Sydney, Sydney, New South Wales, Australia; ${ }^{3}$ Kolling Institute, Northern Clinical School, University of Sydney, Sydney, New South Wales, Australia

Correspondence to Professor Gin S Malhi, Department Head and Chair of Psychiatry, Advanced Research High-field Imaging Facility, Kolling Institute, Clinical and Research Director CADE Clinic, Level 3, Main Hospital Building, Royal North Shore Hospital, St. Leonard's, Sydney, NSW 2065, Australia; Gin.malhi@sydney.edu.au

\begin{abstract}
The term treatment-resistant depression (TRD) is widely used in the context of managing mood disorders, but defining it, both conceptually and in practice, has proven difficult. Most definitions have focused on pharmacotherapy but even these have struggled to capture the complexity of varying response and duration of treatment. Both clinically and for research studies a meaningful definition of TRD is necessary because it may lead to the development of 'therapy-defined depressive subtypes' and the discovery of novel antidepressants. This brief perspective critically reviews the concept of treatment resistance and how it can be more clearly defined so as to achieve a better understanding of depression and facilitate clinical treatment trials.
\end{abstract}

\section{INTRODUCTION}

Every so often there is a resurgence of interest in treatment-resistant depression (TRD) - a term that first appeared in the literature in the 1970s and has since supplanted refractory depression as the usual descriptor for 'non-response to treatment'. These recurring bursts of enthusiasm are usually triggered by the prospect of new or improved treatments for tackling depression. For example, in recent years, brain stimulation strategies such as ketamine, ${ }^{1}$ stimulants $^{2}$ and physical therapies of various kinds, ${ }^{3-5}$ are increasingly being used to treat depression-even though evidence for long-term clinical efficacy and sustained functional benefits is yet to be established.

With every new wave of hopeful therapies, concerns regarding the definition and impact of TRD are expounded, but in doing so the concept is reified. Usually this entails outlining the growing prevalence of TRD and lamenting its clinical ${ }^{6}$ and financial costs; ${ }^{7}$ thus constructing a need for new treatments and justifying experimentation. However, by definition, as successive novel treatments fail to make an impression, TRD persists, reflecting its true nature as a chronic, unremitting illness that manifests as residual depressive symptoms and eventuates in high levels of disability and mortality. ${ }^{8}$

Therefore, the important clinical considerations are: Is TRD conceptually valid? And if so, how can we define treatment resistance more meaningfully?

\section{THE CONCEPT OF TRD: WHAT IS IT?}

A common definition of TRD is an 'inadequate response to adequate antidepressant therapy'. ${ }^{9}$ There are two parts to this definition: response (the inverse of resistance) and antidepressant therapy (which can be of many kinds).

\section{Response}

Response is an important concept for both clinicians and patients and can be usefully defined as a 'clinically meaningful reduction in symptoms that leads to functional recovery'. ${ }^{10-12}$ In practical terms, response is used to gauge decision-making such as when to modify antidepressant dosage, augment therapy, combine medications or substitute them altogether, and switch strategy. ${ }^{10}{ }^{11}$ In research, response is conventionally a reduction in baseline symptomatology of $50 \%$ or more, as measured on standard depression rating scales such as the HAM-D or MADRS. This means that those that do not achieve a $50 \%$ reduction in their score are deemed treatment resistant, and in practice, a significant proportion of depressed patients - who only achieve a partial response to treatment-qualify. Thus on this basis TRD includes those who have 'no response' to treatment whatsoever and those that have a 'partial response'. Furthermore no real distinction is drawn between the various symptoms of depression-meaning that a quantitatively similar response can reflect improvement across very different symptom domains. For example, improvement in one set of symptoms such as anxiety, fatigue and sleep is 'equivalent' to improvement in a separate set of symptoms such as suicidal thinking, guilt and psychomotor slowing. However, these groups of depressive symptoms are clinically very different and therefore distinguishing subgroups of response is important because they likely reflect different kinds of treatment resistance (see figure 1).

As the symptoms of depression diminish and depression resolves, treatment response melds into remission. However, a complete absence of depressive symptoms is uncommon and at least $50 \%$ of 'remitted' patients continue to experience at least two or more symptoms. ${ }^{13}$ These residual symptoms, also described as 'subsyndromal', usually involve neurovegetative symptoms such as changes in sleep and appetite, and are important because their presence increases the likelihood of relapse or recurrence. However, if remission is sustained it transmutes to recovery - accompanied by functional improvement. Thus, in reality, response is dimensional with no clear-cut trend breaks or steps. This means that resistance (the inverse of response) is also dimensional-especially if measured using number and severity of depressive symptoms. This is a fundamental problem because the management of depression involves step-wise administration of treatments. The dose of a medication or frequency of psychotherapy can be altered but the decision to pursue a particular treatment strategy is dichotomous (yes or no). This is why most models of TRD involve staging. ${ }^{9}{ }^{14-17}$

\section{Antidepressant therapy}

Definitions of TRD focus predominantly on failures of pharmacotherapy and physical treatments, with only modest consideration given to outcomes following psychological therapies and social interventions; perhaps because these can be more reliably quantified. However, even the most sophisticated and seemingly accurate definitions ${ }^{914}$ of TRDprove difficult to apply to real-world clinical patients. In part this is a consequence of the treatment target (depressive symptoms) being amorphous, which makes it difficult to assign a fixed and clinically meaningful threshold for treatment response. This variability in defining treatment response/resistance is reflected in the differing approaches adopted by models of TRD, and in practice, this translates to the use of an assortment of treatment strategies and paradigms. A wide range of antidepressants offers choice, and each can be further varied in terms 


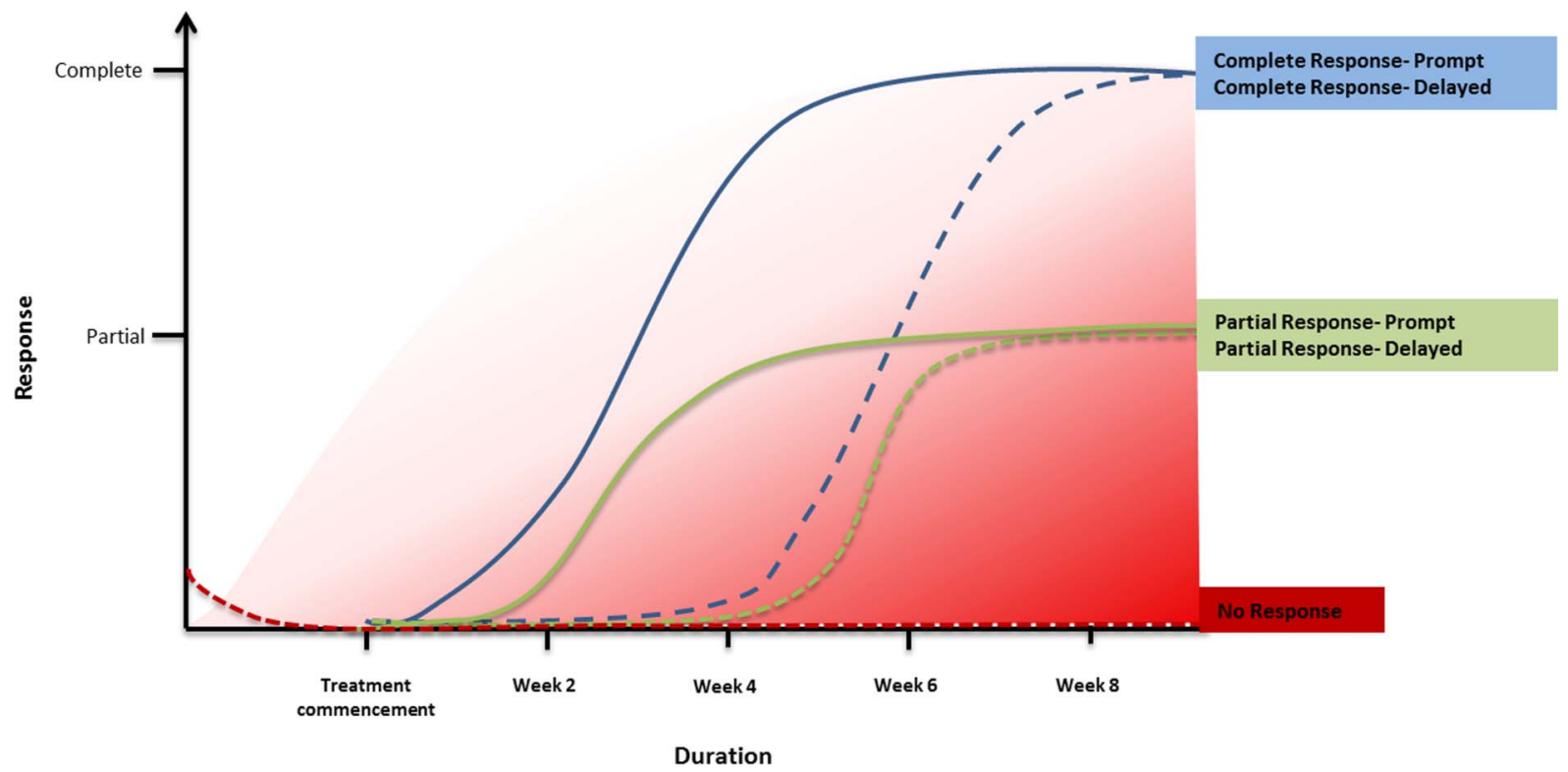

Figure 1 Important aspects of antidepressant treatment are the timing and pattern of response. Debate concerning the duration of antidepressant treatment reflects uncertainty as to when response typically occurs, if it emerges at all. The speed of response varies considerably, as does its extent-with the majority of individuals experiencing a delay and/or only a partial response. It is important to note that rapid responders may be a different group to slow responders-forming perhaps another type (degree) of treatment-resistant depression (TRD). Similarly those that respond only partially may be regarded as another subtype of TRD. In the figure response (y axis) is charted according to time ( $x$ axis). The various lines indicate potential response trajectories. The blue lines indicate complete response, the green lines indicate partial response and the red line indicates no response. The dashed lines denote a delay in response. The graded red shading indicates degree of treatment resistance, which increases as the time to a response increases and the extent of the response decreases.

of dosing and duration of administration. This makes determining TRD difficult.

For instance, a common definition of TRD is that of an inadequate response to at least two adequate successive antidepressant treatments. The accurate application of this definition may provide a useful clinical tool for classifying depressed patients who are truly treatment resistant; but there are several inherent methodological limitations. First and foremost the term 'adequate', that is used to benchmark antidepressant treatment, actually refers to the overall 'quality' of management. It encompasses a range of additional factors that influence treatment response, including whether the patient is accepting of the diagnosis and the need for treatment. These factors are important because they determine treatment compliance, and like antidepressant therapy parameters they vary according to type of depression. Hence treatment adequacy is a complicated concept, but most clinical guidelines consider an antidepressant trial to be adequate if the agent is administered for at least 3 weeks at the optimal dose. It is noteworthy that here too there is considerable variation in duration and dose; for example, a patient treated with a standard antidepressant dose for 4 weeks is essentially 'equal' to another patient prescribed much higher doses for a longer period of time. Both are deemed to have 'trialled the medication adequately' - and if they fail to respond are rated as treatment resistant to the same extent. Thus both the dose and duration of antidepressant treatment, though of critical importance, are often not captured with sufficient precision in clinical practice to define TRD, and therefore the key clinical question-when should treatment nonresponse be regarded as treatment resistance, remains unanswered.

\section{WHEN DOES NON-RESPONSE BECOME TREATMENT RESISTANCE?}

The few longitudinal sequential antidepressant treatment studies that have been conducted show that usually three antidepressant trials are needed before the majority of patients respond to the point of remission. ${ }^{18} 19$ Therefore, non-response, which includes partial responses, is the most likely outcome. Trying to operationalise the type of antidepressant treatment, its dose and duration is, as we have seen, difficult and produces variable definitions. An alternative approach, that may be of some practical use, is to consider what treatment resistance means from different perspectives. For example, patients regard resistance to treatment as 'not getting back to their usual self'. This seemingly straightforward definition encompasses restoration of normal functioning, and though of course it is a reasonable expectation, in reality, it is often difficult to achieve. In contrast, doctors apply a relatively modest threshold for adequacy of response and are usually satisfied with some discernible improvement in symptoms and a return of some degree of functioning. In other words, patients aim for recovery whereas doctors aim for remission and therefore their respective views on non-response and treatment resistance usually differ. ${ }^{18} 19$

However, for both patients and their treating doctors the successive failure of treatment strategies to achieve an adequate response (meeting their respective goals of remission or recovery) leads to the realisation of treatment resistance.

This change in perception and the fact that the views of patients and doctors often differ is important because the change in perspective signifies openness to consideration of new and alternative treatment strategies. In these instances, assuming the diagnosis and formulation of depression are correct and the appropriate paradigm of treatment is being employed, a depressive illness not responding to suitable treatment suggests that the 'illness' is either too severe, or of a different kind altogether (eg, bipolar depression).

\section{MEANINGFULLY DEFINING TREATMENT RESISTANCE}

Defining TRD allows partitioning of the depressive population into treatment sensitive (and non-responsive) groups. Those that do not respond to a particular treatment form a useful group within which to trial new and alternative agents. Enrichment of samples for treatment trials in this manner would ensure that more homogeneous samples of patients are investigated. It would also replicate real-world practice where some 
medications, for example, selective serotonin reuptake inhibitors are usually prescribed first line but if they fail remarkably little is known about which antidepressant to prescribe next. In this scenario TRD arises following the very first instance of treatment non-response and includes all patients that fail an adequate trial of first-line medication. Parameters used to define TRD include clinical history: number of previous episodes and their duration (a proxy of disease burden), previous history of response or non-response, family history and comorbid illness. In addition examining the symptom profile of those that respond and do not respond more closely - and the extent to which certain features are treated, can also help to further refine TRD. For example, subtypes of TRD could include those that are responsive overall, but have no (or little) response in a particular domain of symptoms, such as cognition or vegetative symptoms. This approach would introduce the concept of specificity of response and focus attention on defining response according to particular clinical domains or symptoms.

\section{CONCLUSION}

Currently, at some point in the course of their illness, the majority of depressed patients experience a poor response to antidepressants, and many will end up having TRD. Therefore, researching TRD is imperative but despite the significant benefits of targeting TRD there are also some important limitations that need to be borne in mind.

Potential benefits of clinical research in TRD patient populations:

1. The definition and recognition of TRD serves as a useful reminder that current antidepressant treatments for depression are far from ideal.

2. Defining TRD systematically on the basis of antidepressant nonresponse may provide 'therapy-defined depressive subtypes', which represent patient groups non-responsive to certain treatments. TRD-enriched populations would be smaller and more homogeneous and could be used to trial more ambitious treatments such as combinations, augmentation strategies and newer therapies and even those that are experimental.

Important limitations of clinical research in TRD patient populations:

1. Testing new antidepressants in patients with TRD means that the development of first-line antidepressant therapies may be limited.

2. The threshold for response in TRD is higher and therefore new antidepressants have to be especially effective to achieve discernible improvement. This means the bar (which needs to be raised) may in actual fact be set too high. This would mean that potentially effective antidepressants may be overlooked because they fail in trials conducted exclusively in TRD-enriched samples.

In sum, it is important to note, that agents in our current armamentarium of antidepressants only ever achieve symptomatic improvement and that none of the medications are in reality curative; antidepressants simply facilitate recovery. ${ }^{20}$ Furthermore, in some cases, TRD is actually 'refractory' and that some kinds of depression may in fact lie beyond our reach in terms of offering satisfactory treatment.

Competing interests None declared.
Provenance and peer review Commissioned; externally peer reviewed. doi:10.1136/eb-2015-102299

Accepted 23 December 2015

\section{REFERENCES}

1. Caddy C, Amit BH, McCloud TL, et al. Ketamine and other glutamate receptor modulators for depression in adults. Cochrane Database Syst Rev 2015;9:CD011612

2. Lavretsky H, Reinlieb M, St Cyr N, et al. Citalopram, methylphenidate, or their combination in geriatric depression: a randomized, double-blind, placebo-controlled trial. Am J Psychiatry 2015;172:561-9.

3. Dunner DL, Aaronson ST, Sackeim HA, et al. A multisite, naturalistic, observational study of transcranial magnetic stimulation for patients with pharmacoresistant major depressive disorder. J Clin Psychiatry 2014;75:1394-401.

4. Dougherty DD, Rezai AR, Carpenter LL, et al. A randomized sham-controlled trial of deep brain stimulation of the ventral capsule/ventral striatum for chronic treatment-resistant depression. Biol Psychiatry 2015;78:240-8.

5. Kayser S, Bewernick BH, Matusch A, et al. Magnetic seizure therapy in treatment-resistant depression: clinical, neuropsychological and metabolic effects. Psychol Med 2015;45:1073-92.

6. Fostick L, Silberman A, Beckman M, et al. The economic impact of depression: resistance or severity? Eur Neuropsychopharmacol 2010;20:671-5.

7. Ivanova JI, Birnbaum HG, Kidolezi Y, et al. Direct and indirect costs of employees with treatment-resistant and non-treatment resistant major depressive disorder. Curr Med Res Opinion 2010;26:2475-84

8. Fekadu A, Wooderson SC, Markopoulo K, et al. What happens to patients with treatment-resistant depression? A systematic review of medium to long term outcome studies. J Affect Disord 2009;116:4-11.

9. Fava M. Diagnosis and definition of treatment-resistant depression. Biol Psychiatry 2003:53:649-59.

10. Rush AJ, Kraemer HC, Sackeim HA, et al. Report by the ACNP Task Force on response and remission in major depressive disorder. Neuropsychopharmacol 2006;31:1841-53.

11. Malhi GS, Bassett D, Boyce $P$, et al. Royal Australian and New Zealand College of Psychiatrists clinical practice guidelines for mood disorders. Aust New Zeal J Psychiatry 2015;49:1087-206.

12. Murray G. You say you want a revolution: recovery, biomedicine and muddling through. Aust New Zeal J Psychiatry 2015;49:1085-6.

13. Nierenberg $\mathbf{A}$, Husain $M$, Trivedi $M$, et al. Residual symptoms after remission of major depressive disorder with citalopram and risk of relapse: a STAR ${ }^{*} D$ report. Psychol Med 2010;40:41-50.

14. Thase ME, Rush AJ. When at first you don't succeed: sequential strategies for antidepressant nonresponders. J Clin Psychiatry 1997;58:23-9.

15. Souery D, Amsterdam J, de Montigny C, et al. Treatment resistant depression: methodological overview and operational criteria. Eur Neuropsychopharm 1999:9:83-91.

16. Oquendo MA, Baca-Garcia E, Kartachov A, et al. A computer algorithm for calculating the adequacy of antidepressant treatment in unipolar and bipolar depression. J Clin Psychiatry 2003;64:825-33.

17. Fekadu A, Wooderson S, Donaldson C, et al. A multidimensional tool to quantify treatment resistance in depression: the Maudsley staging method. J Clin Psychiatry 2009;70:177-84.

18. Warden D, Rush AJ, Trivedi $\mathrm{MH}$, et al. The STAR* D Project results: a comprehensive review of findings. Curr Psychiatry Rep 2007;9:449-59.

19. Rush AJ, Trivedi MH, Wisniewski SR, et al. Acute and longer-term outcomes in depressed outpatients requiring one or several treatment steps: a STAR ${ }^{*} D$ report. Am J Psychiatry 2006;163:1905.

20. Harmer CJ, Goodwin GM, Cowen PJ. Why do antidperssants take so long to work? A cognitive neuropsychological model of antidepressant drug action. Br J Psychiatry 2009;195:102-8. 